\title{
Traducción y adaptación transcultural al español del Simulator Sickness Questionnaire \\ Translation and cross-cultural adaptation to Spanish of the Simulator Sickness Questionnaire
}

\author{
Pablo Campo-Prieto, Gustavo Rodríguez-Fuentes, José Ma Cancela-Carral \\ Universidad de Vigo (España)
}

\begin{abstract}
Resumen. La Realidad Virtual (RV) es una herramienta cada vez más presente en la valoración y tratamiento del paciente neurológico. Sin embargo, en ocasiones, la exposición a entornos inmersivos puede desencadenar efectos adversos. Detectar las posibles complicaciones de su uso se antoja fundamental para dotar de seguridad a sus aplicaciones terapéuticas. El objetivo de este trabajo ha sido realizar una traducción y adaptación transcultural del cuestionario Simulator Sickness Questionnaire (SSQ), empleado para valorar el cybersickness o sintomatología adversa asociada al uso de la RV, de cara a minimizar riesgos vinculados a su uso en población española. Se llevó a cabo una traducción, retrotraducción y adaptación del instrumento original y una validación de contenido y equivalencia semántica del cuestionario a través de la opinión de expertos, llegando a una versión preliminar evaluada en un ensayo piloto con 54 participantes. Tras la obtención de la versión preliminar en español, el análisis de validez de contenido mostró valores elevados (índice de validez de contenido 0,89; valores de kappa 0,89). 42 de los 54 participantes en el estudio (78\%) entendieron el total de la equivalencia semántica planteada y el 100\% de la muestra comprendió 13 de los 16 ítems adaptados (81\%). El proceso de traducción y adaptación transcultural al castellano del SSQ llevado a cabo, ha dado como resultado una versión equivalente al cuestionario original, presentando una elevada concordancia semántica que facilitará el desarrollo de futuros estudios en población española sobre la sintomatología adversa que pudiera generar la RV.
\end{abstract}

Palabras clave: realidad virtual, síntomas, evaluación, neurología, actividad física.

\begin{abstract}
Virtual Reality (VR) uses is growing in the assessment and treatment of neurological patients. However, immersive environments exposure can trigger adverse effects. Checking the possible complications of its use is essential to provide security to therapeutic approaches. The aim of this work has been to carry out a translation and cross-cultural adaptation of the Simulator Sickness Questionnaire (SSQ), used to assess cybersickness or adverse symptoms associated with VR, with the aim to decrease risks in the Spanish population. Forward-translation, back-translation and cross-cultural adaptation of the original questionnaire was carried out. Content validation and semantic equivalence was assessed by an expert panel, leading to a preliminary version evaluated in a pilot trial with 54 participants. After obtaining the preliminary version in Spanish, the content validity analysis showed high values (content validity index 0,89 and kappa values 0,89). 42 of the 54 participants (78\%) understood the total semantic equivalence raised and $100 \%$ of the sample understood 13 of the 16 adapted items $(81 \%)$. The translation and cross-cultural adaptation into Spanish of the SSQ was carried out in this study, showed an equivalent version to the original questionnaire, presenting a high semantic concordance and facilitating the development of future studies in the Spanish population on the adverse symptoms that VR could generate.
\end{abstract}

Keywords: virtual reality, symptoms, assessment, neurology, physical activity.

\section{Introducción}

La Realidad Virtual (RV) es una herramienta interactiva, inmersiva y realista fundamentada en un entorno tridimensional que genera un mundo virtual utilizando un hardware que permite a los usuarios vivir una experiencia en primera persona en un mundo virtual (Benham, Kang \& Grampurohit, 2019; Davis,

Fecha recepción: 08-02-21. Fecha de aceptación: 21-07-21

Jose $\mathrm{M}^{\mathrm{a}}$ Cancela Carral

chemacc@uvigo.es
Nesbitt \& Nalivaiko, 2014).

En los últimos años se ha vuelto muy popular (Yildirim, 2020) y, dispositivos como los head-mounted display (HMD) han sido utilizados en aplicaciones de ámbitos tan distintos como educación, entretenimiento, ingenierías, simuladores de conducción y vuelo, y también en el campo de la salud (Davis et al., 2014; Duque et al., 2013; Rebenitsch \& Owen, 2016).

En este último ámbito, el paciente neurológico se ha beneficiado de esta novedosa herramienta. Así, se ha podido comprobar su uso en patología post ictus (Bank, Cidota, Ouwehand \& Lukosch, 2018) Enfermedad de 
Parkinson (Kim, Darakjian \& Fnley, 2017) o dolor de miembro fantasma (Murray et al., 2007) entre otros. Además, estos usos han abarcado tanto la evaluación de la propia patología y sus secuelas (Davison, Deeprose \& Terbeck, 2018), como el posterior tratamiento rehabilitador (Campo-Prieto, Cancela-Carral, Machado \& Rodríguez-Fuentes, 2021; León-Ruiz, Pérez-Nieves, Arce-Arce, Benito-León \& Ezpeleta-Echávarri, 2019).

Sin embargo, en algunos casos, los sujetos pueden experimentar síntomas desagradables como náusea, desorientación, dolor de cabeza o, en casos extremos, vómitos (Kennedy, Drexler \& Kennedy, 2010). A este conjunto de síntomas se le conoce como cybersickness, el cual se debe, probablemente, a incongruencias y conflictos sensoriales entre estímulos visuales, vestibulares y de movimiento en los entornos virtuales que no se corresponden con el entorno real (Carvalho, Costa \& Nardi, 2011).

Uno de los potenciales peligros de este efecto adverso es que puede desarrollarse durante la exposición, después de horas o incluso días (LaViola, 2000). Bajo estas circunstancias se hace necesario tener un instrumento que pueda evaluar estos aspectos, siendo el Simulator Sickness Questionnaire (SSQ) (Kennedy, Lane, Berbaum \& Lilienthal, 1993) una herramienta ampliamente utilizada en investigaciones sobre cybersickness (Lo \& So, 2001).

El SSQ fue desarrollado por Kennedy et al. (1993) adaptado directamente desde el Pensacola Motion Sickness Questionnaire (Kellogg, Kennedy \& Graybiel, 1965; Kennedy, Tolhurst \& Graybiel, 1965) (MSQ), el cual describía el malestar general a través de la evaluación de un listado de 25 a 30 síntomas. Posteriormente, y para su aplicación en RV, se eliminaron varios ítems, resultando un total de 16 que representan otros tantos síntomas englobados en tres dimensiones (Oculomotor, Desorientación y Náuseas) (Stone, 2017).

De esta versión original se han realizado algunas traducciones a otros idiomas, como la realizada al portugués por Carvalho et al. (2011). También parece haber traducciones a francés (Bouchard, Robillard \& Renaud, 2007) o alemán(Hosch, 2018). Sin embargo, hoy en día, existe un vacío en la adaptación a otros idiomas, entre ellos el castellano, idioma que hablan, según datos (Gayá, 2017), sobre 572 millones de personas. Estos datos apoyan que sea de relevancia que escalas como la SSQ puedan contar con su versión en este idioma.

El número de usuarios de RV en España ha seguido un crecimiento exponencial, fundamentalmente debi- do al sector del ocio, el más desarrollado y pujante, encargado de liderar la financiación a la investigación en el campo de la RV. Esta gran inversión en el sector del entretenimiento ha permitido que otros ámbitos científicos como el médico-terapéutico o el de educación acompañen en esta tendencia al alza (Aznar, Romero y Rodríguez, 2018; Sánchez-Cabrero et al., 2020). Este incremento, desde la perspectiva de salud, también lleva aparejado la preocupación por el posible cybersickness, por lo que se hace necesario su valoración, siendo el SSQ una posible herramienta para ello.

Por lo tanto, el objetivo de este estudio es traducir y adaptar el SSQ al español.

\section{Material y métodos}

\section{Diseño}

Este estudio se ha llevado a cabo tomando como referencia la metodología aceptada internacionalmente propuesta por Beaton, Bombardier, Guillemin \& Ferraz (2000), Guillemin, Bombardier \& Beanton (1993) y Guillemin (1995), la traducción al español de una escala de traumatología realizada por Ares et al. (2013), las directrices de la OMS para estos procesos (WHO, 2016) y la traducción del propio SSQ al portugués llevada a cabo por Carvalho et al. (2011).

\section{Objetivo}

El objetivo de este trabajo ha sido realizar una traducción del SSQ y comprobar si la versión preliminar obtenida es comprendida y aplicable a población general española con diferentes niveles de educación, para poder minimizar los posibles riesgos derivados de la exposición a entornos inmersivos y posteriormente adecuar las tareas a la modalidad más apropiada.

\section{Participantes}

La muestra estuvo formada por un total de 54 participantes adultos que fueron seleccionados de una muestra de conveniencia con diferente género, edad y nivel educativo para garantizar la comprensión del cuestionario por una muestra representativa de la población general española (ver tabla 1).

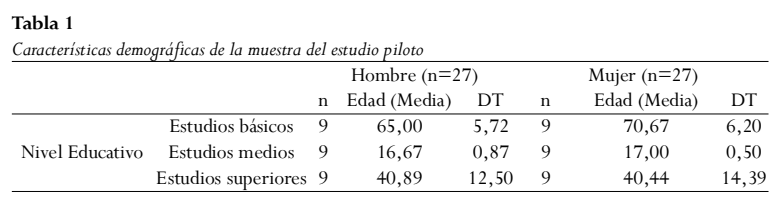

\section{Procedimiento}

El SSQ es una herramienta ampliamente utilizada 
en investigaciones sobre cybersickness (Lo \& So, 2001), la cual se compone de 16 ítems. Cada uno de los ítems hace referencia a un posible síntoma desencadenado por el uso de un entorno de RV. Los síntomas son puntuados a través de una escala de Likert de cuatro niveles ( 0 , ausente; 1 , leve; 2, moderado; 3, grave). Estos 16 síntomas se agrupan en tres dimensiones (Oculomotor, Desorientación y Náuseas). La puntuación de cada una de las dimensiones se obtiene a través de las puntuaciones específicas de cada uno de los ítems, los cuales deben ser sumados y, por último, colocados en una fórmula de conversión. El marcador general del SSQ se obtiene tras aplicar los valores finales de cada dimensión en un algoritmo específico (Kennedy et al., 1993).

El proceso de traducción y adaptación transcultural del SSQ siguió los protocolos estandarizados (Beaton, Bombardier, Guillemin \& Ferraz, 2000) y los resultados de la evaluación del cuestionario SSQ puede observarse en la Figura 1.

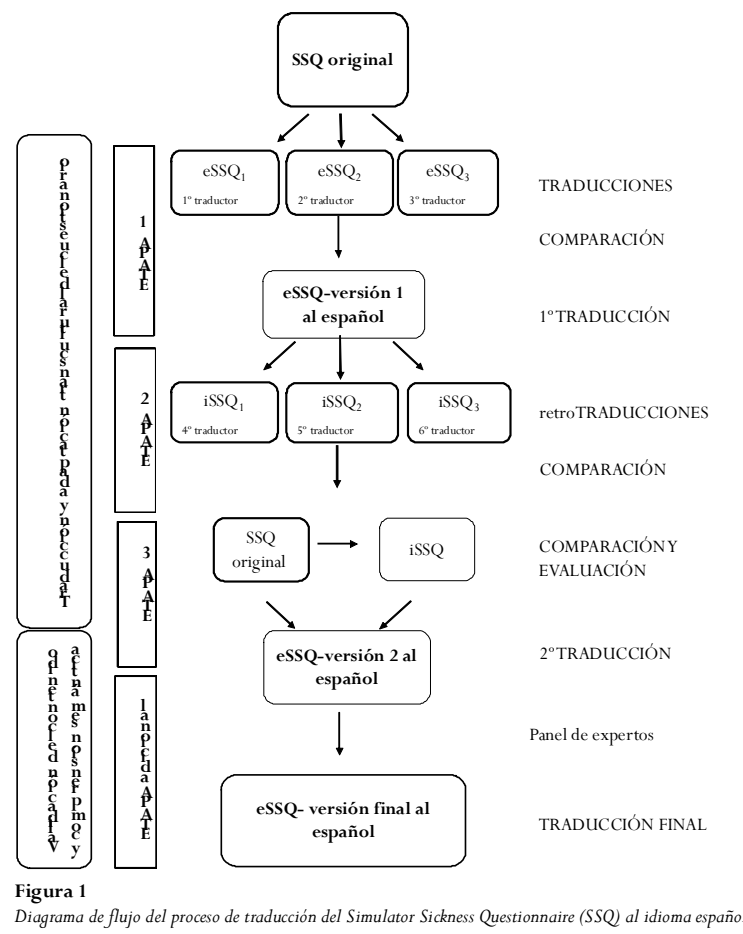

La metodología aplicada se resume en las siguientes etapas:

Etapa 1: Triple traducción del cuestionario original (inglés) al español, por tres traductores profesionales (inglés nativo), que realizaron sus traducciones cegados y de manera independiente. Se les pidió que prestasen especial atención a la consistencia semántica de los términos. Tras una comparativa se obtuvo una primera versión de SSQ en español.

Etapa 2: Triple retrotraducción al inglés por tres pro- fesoras españolas del Grado Traducción e Interpretación de la Universidad de Vigo especialistas en inglésespañol, de manera independiente, cegada y sin conocimiento previo de la escala original.

Etapa 3: Comparación y evaluación de la equivalencia semántica entre la versión original del cuestionario y el obtenido tras la retrotraducción, llevado a cabo por los autores de este estudio (PC, JMC y GR) y un médico investigador bilingüe experto (ME), acostumbrado a utilizar cuestionarios médicos. Después de este proceso se obtuvo una segunda versión española del SSQ.

Etapa 4: Teniendo en cuenta que el SSQ debe medir sintomatología provocada por entornos virtuales e inmersivos, se seleccionó un panel de expertos atendiendo a su carrera profesional, académica y experiencia laboral en el ámbito de la ingeniería informática, programación de videojuegos y gaming. Se les pidió que evaluasen la claridad del contenido para cada ítem mediante una escala tipo Likert de 5 puntos $(0=$ no está claro a $4=$ muy claro) (Polit, Beck \& Owen, 2007).

La validez de contenido del cuestionario para la claridad (tabla 2), se estimó a través de los índices de validez de contenido para cada ítem (I-CVI, calculado como la razón entre el número de expertos que habían dado una puntuación de 3 o 4 en la escala Likert y el número total de expertos que había participado) y para toda la escala (S-CVI, calculado como la razón entre la suma de los I-CVI de todos los ítems y el número total de ítems del instrumento) (Polit et al., 2007). Además, se calculó el índice kappa modificado (k) para eliminar el posible acuerdo por azar entre los expertos. (Sousa \& Rojjanasrirat, 2001; Vega \& Gonzalez, 2020).

\begin{tabular}{lccl}
$\begin{array}{l}\text { Tabla } 2 \\
\text { Versión española del Simulator Sickness Questionnaire (SSQ) }\end{array}$ & & \\
\hline \multicolumn{1}{c}{ Versión española } & I-CVI & $\begin{array}{c} \\
\mathrm{k}\end{array}$ & Evaluación \\
\hline $\begin{array}{l}\text { 1. Malestar general } \\
\text { 2. Cansancio }\end{array}$ & 1 & 1 & $\begin{array}{l}\text { Excelente } \\
\text { 3. Dolor de cabeza }\end{array}$ \\
4. Vista cansada & 1 & 1 & $\begin{array}{c}\text { Excelente } \\
\text { Excelente }\end{array}$ \\
5. Dificultad para enfocar & 0,994 & 1 & Excelente \\
6. Aumento de salivación & 0,898 & 0,992 & Excelente \\
7. Sudoración & 0,994 & 0,996 & Excelente \\
8. Náusea & 1 & 1 & Excelente \\
9. Dificultad para concentrarse & 1 & 1 & Excelente \\
10. Pesadez de cabeza & 1 & 1 & Excelente \\
11.Visión borrosa & 0,898 & 0,896 & Excelente \\
12. Mareos con ojos abiertos & 1 & 1 & Excelente \\
13. Mareos con ojos cerrados & 1 & 1 & Excelente \\
14. Vértigo & 1 & 1 & Excelente \\
15. Estómago revuelto & 1 & 1 & Excelente \\
16. Eructos & 1 & 1 & Excelente \\
\hline & 1 & 1 & Excelente \\
\hline
\end{tabular}

Para finalizar, se realizó un estudio piloto, el objetivo fue evaluar la comprensión de los ítems propuestos en la versión al castellano y, de ser el caso, utilizar las sugerencias u opiniones obtenidas para mejorar la ver- 
sión definitiva del cuestionario. Con la muestra seleccionada se trató de obtener el mayor valor ecológico posible diversificando a conveniencia a los sujetos participantes: hombres y mujeres con edades comprendidas entre 15-79 años con diferente nivel de educación general y que hubiesen probado un simulador en alguna ocasión.

Los sujetos participantes fueron divididos en tres grupos atendiendo a su nivel de educación: Grupo estudios básicos (EGB, ESO, FP de grado básico), Grupo estudios medios (Bachillerato o FP de grado medio) y Grupo estudios superiores (Universitario). Se les pidió que cumplimentasen el formulario contestando si cada ítem estaba claro y era entendible o, en su defecto, si tenían alguna sugerencia para mejorar la comprensión de algún ítem del cuestionario.

Considerando los resultados y sugerencias obtenidas tras las respuestas, se elaboró la versión española final del SSQ. El proceso completo se resume en la Figura 1.

Por otra parte, se solicitó y recibió la aprobación del Comité de Ética de la Facultad de Fisioterapia de la Universidad de Vigo (205/2019_1) y se respetaron los acuerdos de la Declaración de Helsinki. Todos los participantes aceptaron y firmaron un consentimiento informado.

\section{Resultados}

La escala original, las distintas traducciones (T1, T2 y T3), las respectivas retrotraducciones (R1, R2 y R3) y la versión final en castellano del cuestionario SSQ se muestran en la Tabla 3.

La versión revisada por los traductores mostró algunas diferencias entre ellos en relación con los términos propuestos, pero en otros la coincidencia fue total. Así, en los ítems 3, 7, 8, 9, 11, 12, 13 y 14 hubo una coincidencia total entre sí. No tanto en relación con los ítems 1, 2, 4, 6 y 16, donde se encontraron pequeñas diferencias en los términos traducidos, pero que no provocaron una alteración semántica. Las principales diferencias se dieron en los ítems 5, 10 y 15. En el ítem 5, «Difficulty focusing», se le atribuyeron dos traducciones distintas, T1: dificultad para enfocar, mientras T2 y T3: dificultad para concentrarse. Estas dos últimas traducciones fueron coincidentes con las obtenidas en el ítem 9, «Difficulty concentrating». En el ítem 10, «Fullness of the head», las traducciones fueron, T1: Saturación, T2: Saturación cognitiva y T3: Pesadez de cabeza. En el ítem 15, «Stomach awareness», las traducciones fueron T1 y T2: Sensibilidad estomacal yT3: Ma- lestar de estómago. Con respecto a las Retrotraducciones se dieron coincidencias en los ítems 2, 3, 4, 7, 8, 9, 11, 12, 13, 14 y 16; hubo también retrotraducciones similares en los ítems 1, 5 y 6 . Las principales divergencias se observaron en los ítems 10 y 15, debido a la dificultad para dar equivalencia al sentido de las expresiones anglosajonas y que fueron obtenidas tras la traducción literal del primer término de la escala original y dando como resultado una retrotraducción al inglés alejado del término original. Comparando las traducciones con la versión original se optó por diferenciar los ítems 5 y 9. Así, aunque en su traducción fueron coincidentes en las traducciones T2 y T3, prevaleció la congruencia con la versión original manteniendo 2 términos independientes. Por su parte, el ítem 15, «Stomach awareness», obtuvo una traducción alejada de su significado, puesto que la traducción literal aportada por T1 y T2: sensibilidad estomacal, fue muy diferente a la de la aportada por T3: malestar de estómago. Este ítem se acerca más al sentido del ítem original en el contexto de la aplica-

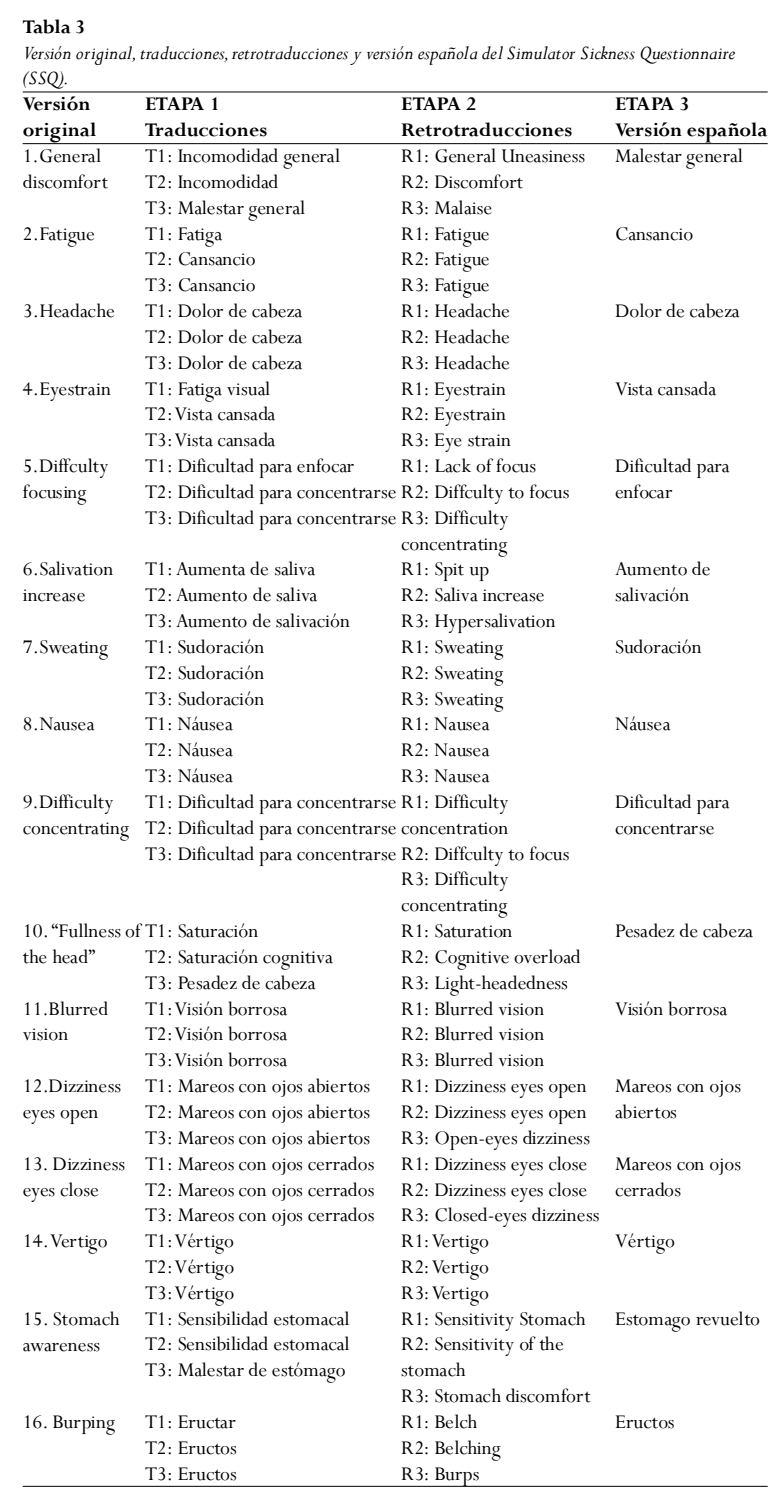


ción del cuestionario, pero teniendo en cuenta que el término «malestar de estómago» puede ser entendido como general y, tras la consideración de ME y las opiniones consensuadas de los autores, se optó por el término «estómago revuelto», más específico y reconocible tras la exposición a determinados simuladores.

De hecho, respecto a la claridad del contenido, el ICVI fue 1,00 para 12 ítems (incluyendo el propio ítem 15), 0,994 para dos ítems y 0,898 para los dos ítems restantes. El S-ICV fue 0,986 (Tabla 1). Los valores de $\mathrm{k}$ fueron calificados como excelentes. Tras la evaluación de los expertos se mantuvo el término «estómago revuelto» para el ítem 15 y se reafirmó la diferenciación entre los ítems «dificultad para enfocar»y «dificultad para concentrarse».

En la versión final, todos los ítems incorporados fueron obtenidos de, al menos, una versión propuesta por los traductores a excepción del ítem 15. En los ítems 1, 2, 3, 5, 6 y 10 se optó por la forma con mayor aproximación literal a su significado en español y que, a su vez, pudiera ser fácilmente comprendida por todo tipo de sujetos. Trece de los dieciséis términos fueron comprendidos por los participantes en el estudio piloto, aunque en los ítems «aumento de saliva», «dificultad para enfocar» o «pesadez de cabeza» algunos participantes (3, 10 y 12 de 54, respectivamente) señalaron no comprender el término, sugiriendo uno de ellos «aumento de salivación» y otro «abotargado», como opciones para «aumento de saliva»y «pesadez de cabeza», respectivamente. El total de los participantes consideró el cuestionario rápido y sencillo de cubrir. Teniendo en cuenta estas opiniones y tras discusión entre los redactores del proyecto, se optó por mantener la versión inicialmente planteada.

\section{Discusión}

El presente estudio es el primero que traduce y adapta el cuestionario SSQ a la lengua castellana. Se ha priorizado la equivalencia semántica de los términos por encima de una traducción literal o demasiado técnica de los mismos. Es importante destacar que debe ser comprendida por todo el estrato de población, independientemente de su nivel sociocultural y educativo. Aun así, el proceso desarrollado cumple con los estándares de calidad propuestos por las recomendaciones generales como la traducción ciega (Beaton et al., 2000; Guillemin et al., 1993). Un punto fuerte de nuestro estudio fue poder contar con tres traductores profesionales y tres profesoras del Grado de Traducción e Inter- pretación de la Universidad de Vigo que actuaron cegadas y sin conocimiento del propósito de las traducciones, además de la colaboración de un médico investigador experto bilingüe (ME), para dotar de mayor consistencia a los síntomas psicofisiológicos descritos. Por otra parte, los autores del estudio son profesionales de la Salud (PC y GR) y de la Educación (JMC y GR) y el panel de expertos formado por profesionales pertenecientes a diversos ámbitos y perfiles vinculados al ámbito del gaming.

La variedad de la muestra permitió testar la viabilidad del cuestionario independientemente del sexo, edad o nivel de educación en población española. El número de participantes $(n=54)$ fue superior al marcado $(n=30$ 40) en las guías recomendadas (Beaton et al., 2000).

La presencia de sintomatología vinculada a la exposición a experiencias inmersivas es inherente a la RV desde su concepción (LaViola, 2000) y se ha de considerar como un importante efecto adverso que puede limitar su uso. Davis et al. (2014), establecieron tres factores como posibles desencadenantes del cybersickness: individuales, del dispositivo y de la tarea. Teniendo en cuenta estas premisas, parece importante poder identificarlo y medirlo: primero por la propia seguridad de los usuarios de RV, segundo para colaborar con el desarrollo de dispositivos con mejoras técnicas y, en tercer lugar, para dotar de más información a los responsables últimos de ofrecer esta tecnología tanto a la población con patología como a la población general, para que repercuta en una mayor seguridad.

El campo de la investigación no debe ser ajeno a estas cuestiones, puesto que, si se quiere avanzar en los distintos ámbitos de aplicación de la RV, es necesario que se lleven a cabo ensayos clínicos que doten de evidencia científica a la aplicación de esta nueva herramienta. Así, algunas investigaciones ya han examinado la relación de puntuaciones altas en el SSQ y síntomas individuales, con mayores porcentajes de abandono en los experimentos con simuladores, por lo que también parece justificado poder medir estos síntomas a fin de que estos ensayos no estén abocados al fracaso. Los principales síntomas señalados, y que condicionan mayormente las pérdidas de participantes, son: náusea, malestar general, estómago revuelto, sudoración, aumento de salivación y vértigo (Balk, Bertola \& Inman, 2013).

Para poder determinar y mensurar esta sintomatología, el SSQ se ha propuesto como la escala de elección y de esta forma, tomando como base la traducción y adaptación de esta herramienta ampliamente utilizada en lengua inglesa (Chang, Kim \& Yoo, 2020), 
se podrán identificar y valorar estos síntomas en población española. De esta manera, se abre una puerta a todos los estudios de RV en el país que quieran evaluar el malestar que pueda desencadenar en los usuarios de la RV en su propio idioma, sobre todo de quienes padecen una patología o una enfermedad, así como dotar de mayor seguridad a las aplicaciones con RV en los contextos clínico, educativo o de perfil investigador.

Debido también a esta necesidad de adaptación a la población diana, en la literatura se han podido consultar otros estudios que han creado versiones de escalas al español adaptadas del inglés y que han seguido procesos similares al seguido en nuestro trabajo. A modo de ejemplo, como la adaptación llevada a cabo por Alonso, Prieto \& Antó (1995), adaptaron el conocido cuestionario de salud SF-36 para ser usado en España, o también Calvo, Sánchez-Pedraza y Peña-Solano (2012), que tradujeron y adaptaron al español la escala SQLS para medir la calidad de vida de pacientes esquizofrénicos, o la adaptación de Guerra-Tapia, Buendía-Eisman \& Ferrando (2018) que, más recientemente, hicieron lo propio para obtener una herramienta en español para el seguimiento de la alopecia androgenética femenina.

Por otro lado, existen algunas limitaciones en este trabajo. Aunque la confección de la muestra se ha llevado a cabo conforme a las recomendaciones de las guías, para obtener el mayor valor ecológico posible, quizás podría haber sido más heterogénea desde el punto de vista de su procedencia geográfica, al haberse circunscrito a una sola región del territorio nacional. Aún así, y teniendo en cuenta los términos semánticos, se considera que la influencia de este aspecto ha sido mínima en el resultado final.

Concluyendo, el proceso de traducción y adaptación transcultural al castellano del Simulator Sickness Questionnaire llevado a cabo en este estudio, ha dado como resultado una versión adaptada al español clara, sencilla y equivalente al cuestionario original, presentando una elevada concordancia semántica y adecuada para detectar y evaluar la posible sintomatología adversa vinculada a la exposición a entornos virtuales. Aunque en futuras investigaciones se deberán acometer sus propiedades psicométricas (validez y fiabilidad), el uso de esta versión obtenida facilitará el desarrollo de futuros estudios en población española que puedan investigar el potencial terapéutico de la RV en diversos tipos de patología, entre ellas la neurológica, detectando y mensurando la sintomatología adversa que se pudiera presentar.

\section{Aplicaciones prácticas}

La traducción y adaptación transcultural al español del Simulator Sickness Questionnaire, permitirá la aplicación del mismo a población española, identificando sintomatología adversa por el uso de aplicaciones de RV.

Debido al aumento exponencial del uso de equipamiento tecnológico vinculado a los videojuegos de diferentes plataformas, se espera que esta herramienta tenga un elevado uso.

\section{Agradecimientos}

La publicación de este trabajo ha sido posible gracias a la Convocatoria de Ayudas de Investigación del CoFiGa 2020-2021. Los autores quieren agradecer al Dr. MariñoEnríquez (ME) por su desinteresada colaboración y ayuda con la interpretación y el consenso de las traducciones y las retrotraducciones obtenidas, así como a los profesionales de la traducción y a las docentes del Grado de Traducción e Interpretación de la Universidade de Vigo por su inestimable colaboración.

\section{Referencias}

Alonso, J., Prieto, L., \& Anto, J. M. (1995). The Spanish version of the SF-36 Health Survey (the SF-36 health questionnaire): an instrument for measuring clinical results. Medicina clínica, 104(20), 771-776.

Ares, O., Castellet, E., Maculé, F., León,V., Montañez, E., Freire,A., ... \& Amillo, J.R. (2013). Translation and validation of 'The Knee Society Clinical Rating System' into Spanish. Knee Surgey Sports Traumatology,Arthroscopy ,21(11), 2618-24.https://doi: 10.1007/ s00167-013-2412-4

Aznar-Díaz, I., Romero-Rodríguez, J.M., \& Rodríguez-García,A.M. (2018). La tecnología móvil de RealidadVirtual en educación: una revisión del estado de la literatura científica en España. EDMETIC, Revista de Educación Mediática y TIC, 7(1), 256-274. https://doi.org/10.21071/edmetic.v7i1.10139

Balk, S.A., Bertola, M.A., \& Inman, V.W. (2013). Simulator sickness questionnaire: twenty years later. En: Proceedings of the 7th International Driving Symposium on Human Factors in Driver Assessment, Training, andVhicle Design:driving assessment 2013, 257-263.

Bank, P.J.M., Cidota, M.A., Ouwehand, P.E.W., \& Lukosch, S.G. (2018). Patient-Tailored Augmented. Reality Games for Assessing Upper Extremity Motor Impairments in Parkinson's disease. Journal of medical systems, 42(12),246. https://doi: 10.1007/ s10916-018-1100-9.

Beaton, D.E., Bombardier, C., Guillemin, F., \& Ferraz, M.B. (2000). Guidelines for the Process of Cross-Cultural Adaptation of SelfReport Measures. Spine (Phila Pa 1976), 25(24), 3186-3191. https: / /doi: 10.1097/00007632-200012150-00014.

Benham, S., Kang, M., y Grampurohit, N. (2019). ImmersiveVirtual Reality for the Management of Pain in Community-Dwelling 
Older Adults. OTJR:occupation participation and health, 39(2), 9096. https: / /doi: 10.1177/1539449218817291.

Bouchard, S., Robillard, G., y Renaud, P. (2007). Revising the factor structure of the simulator sickness questionnaire.Annual Review of CyberTheraphy Telemedicine, 5, 117-122.

Calvo, J.M., Sánchez-Pedraza, R., \& Peña-Solano, D.M. (2012).Traducción y adaptación de la escala SQLS para medir calidad de vida en pacientes con esquizofrenia en Colombia. Revista de la Facultad de Medicina, 60(2), 95-102.

Campo-Prieto, P., Cancela Carral, J. M., Machado de Oliveira, I. \& Rodríguez-Fuentes, G. (2021). Realidad Virtual Inmersiva en personas mayores: estudio de casos. Retos: nuevas tendencias en educación física, deporte y recreación, (39), 1001-1005.doi:https: / / doi.org/10.47197/retos.v0i39.78195

Carvalho, M.R., de Costa, R.T., \& da Nardi,A.E. (2011). Simulator Sickness Questionnaire: tradução e adaptação transcultural. Jornal Brasileiro de Psiquiatría, 60(4), 247-252. http://dx.doi.org/ 10.1590/S0047-20852011000400003.

Chang, E., Kim, H.T., \&Yoo, B. (2020). Virtual Reality Sickness: A Review of Causes and Measurements. International Journal of Human-Computer Interaction, 36(17), 1658-1682.

Davis, S., Nesbitt, K., \& Nalivaiko, E. (2014). A systematic review of cybersickness. In Proceedings of the 2014 conference on interactive entertainment, 1-9. https://doi.org/10.1145/2677758.2677780

Davison, S.M.C., Deeprose, C., \& Terbeck, S. (2018). A comparison of immersive virtual reality with traditional neuropsychological measures in the assessment of executive functions. Acta Neuropsychiatrica, 30(2), 79-89. http://dx.doi.org/10.1017/ neu.2017.14.

Duque, G., Boersma, D., Loza-Diaz, G., Hassan, S., Suarez, H., Geisinger, D.,..\& Demontiero, O. (2013). Effects of balance training using a virtual-reality system in older fallers. Clinical Intervertions in Aging, 8, 257-263. http://dx.doi.org/10.2147/ CIA.S41453.

Gayá, V. (2017). Instituto Cervantes el español por el mundo: es la segunda lengua más estudiada tras el inglés. El siglo de Europa, (1222), 9 .

Guerra-Tapia, A., Buendía-Eisman,A., \& Ferrando, J. (2018).Validación de una adaptación transcultural al idioma español de la escala Hair Specific Skindex-29. Actas Dermo-Sifiliográficas, 109(5), 424-431.

Guillemin, F. (1995). Cross-cultural Adaptation and Validation of Heatth Status Measures. Scandinavian Journal of Rheumatology, 24(2), 61-63. http://dx.doi.org/10.3109/03009749509099285.

Guillemin, F., Bombardier, C., \& Beaton, D. (1993). Cross-cultural adaptation of health-related quality of life measures: Literature review and proposed guidelines. Journal Clinical Epidemiology, 46(12), 1417-1432. http://dx.doi.org/10.1016/08954356(93)90142-n.

Hösch,A. (2018). Simulator Sickness in Fahrsimulationsumgebungendrei Studien zu Human Factors (Doctoral dissertation).

Kellogg, R.S., Kennedy, R.S., \& Graybiel,A. (1965). Motion sickness symptomatology of labyrinthine defective and normal subjects during zero gravity maneuvers. Aerospace Medicine, 36, 315-318.

Kennedy, R. S., Lane, N. E., Berbaum, K. S., \& Lilienthal, M. G. (1993). Simulator sickness questionnaire: An enhanced method for quantifying simulator sickness. The international journal of aviation psychology, 3(3), 203-220. https://doi.org/10.1207/ s15327108ijap0303_3

Kennedy, R.S., Drexler, J., \& Kennedy, R.C. (2010). Research in visually induced motion sickness. Applied Ergonomics, 41(4), 494503. https://doi.org/10.1016/j.apergo.2009.11.006.

Kennedy, R.S., Tolhurst, G.C., \& Graybiel, A. (1965).The effects of visual deprivation on adaptation to a rotating environment. NSAM-918. Research report. Naval School of Aviation Medicine (U.S.), 18, 1-36.

Kim,A., Darakjian, N., \& Fnley, J.M. (2017).Walking in fully immersive virtual environments: an evaluation of potential adverse effects in older adults and individuals with Parkinson's disease. Journal and neuroengineering and rehabilitation, 14(1), 16. http://dx.doi.org/ 10.1186/s12984-017-0225-2.

LaViola, J. J. (2000). A discussion of cybersickness in virtual environments. ACM Sigchi Bulletin, 32(1), 47-56. https:// doi.org/10.1145/333329.333344.

León-Ruiz, M., Pérez-Nieves, M.T., Arce-Arce, S., Benito-León, J., \& Ezpeleta-Echávarri, D. (2019). Evidencias actuales sobre la realidad virtual y su utilidad potencial en la neurorrehabilitación postictus. Revista de Neurolología, 69 (12), 497-506. http:// dx.doi.org/ 10.33588/rn.6912.2019148.

Lo,W.T., \& So, R.H.Y. (2001). Cybersickness in the presence of scene rotational movements along different axes. Applied ergonomics, 32, 1-14. http://dx.doi.org/10.1016/s0003-6870(00)00059-4.

Murray, C.D., Pettifer, S., Howard, T., Patchick, E.L., Caillette, F., Kulkarni, J., \& Bamford, C. (2007). The treatment of phantom limb pain using immersive virtual reality: three case studies. Disability and rehabilitation, 29(18), 1465-1469. http:// dx.doi.org/10.1080/09638280601107385.

Polit, D.F., Beck, C.T., \& Owen, S.V. (2007). Is the CVI an aceptable indicator of content validity? Appraisal and recommendations. Research in Nursing Health, 30(4), 459-467. http: / / dx.doi.org/ 10.1002/nur. 20199.

Rebenitsch, L., \& Owen, C. (2016). Review on cybersickness in applications and visual displays. Virtual Reality, 20(2), 101-125.

Sánchez-Cabrero, R., Novillo-López, M.A., Arigita-García,A., Costa-Román, Ó., Barrientos-Fernández,A., \& Pericacho-Gómez, F.J. (2019). Carencias y limitaciones que afectan al asentamiento de la realidad virtual como tecnología de referencia en la sociedad actual. Revista Espacios, 40(10). 1-7.

Sousa,V.D. , \& Rojjanasrirat,W. (2011).Translation, adaptation and validation of instruments or scales for use in cross-cultural health care research: a clear and user-friendly guideline. Journal of evaluation in Clinical Practice, 17, 268-274. https://doi.org/10.1111/ j.1365-2753.2010. 01434.x.

Stone III, W.B. (2017). Psychometric evaluation of the Simulator Sickness Questionnaire as a measure of cybersickness. Graduate Theses and Dissertations. 15429. https: / / doi.org/10.31274/etd180810-5050

Vega, M. D. L. A. F., \& Lomelí, D. G. (2020). Adaptación al español del cuestionario de autoeficacia para regular el ejercicio. Retos: nuevas tendencias en educación física, deporte y recreación, (38), 595 601.doi: https://doi.org/10.47197/retos.v38i38.75225

World Health Organization (2016). Official WHO process of translation and adaptation of research instruments. 2016. Disponible en: http://www.who.int/substance_abuse/ research_tools/translation/en/

Yildirim, C. (2019). Don't make me sick: investigating the incidence of cybersickness in commercial virtual reality headsets. Virtual Reality, 24, 231-239. https://doi.org/10.1007/s10055-01900401-0. 